\title{
The GH/IGF-I axis and longevity
}

Martin Holzenberger

Inserm U515, Hôpital Saint-Antoine, 184 rue Fbg St-Antoine, 75571 Paris 12, France

(Correspondence should be addressed to Martin Holzenberger; Email: holzenberger@st-antoine.inserm.fr)

\begin{abstract}
Several converging lines of evidence obtained over the last years in a wide variety of experimental model organisms suggest that the ageing process is regulated by genes that encode proteins from the somatotroph axis: longevity genes like daf-2, which were identified using mutant Caenorhabditis elegans strains, turned out to be orthologues of the mammalian genes encoding insulin-like signalling cascade proteins. Transgenic flies with mutations in the corresponding insect genes showed a similar pattern of increased lifespan. Finally, mice with spontaneous mutations leading to pituitary hormone deficiency significantly outlived controls. While these and other genetic models suggest that the downregulation of the somatotroph axis can slow the ageing process, other results from studies using pharmacological administration of growth hormone suggest that such stimulating treatment can restore some of the phenotypic traits associated with youth. To better understand the role of the insulin-like receptors in mammalian lifespan regulation and ageing, we explored the phenotype of heterozygous IGF-I receptor (IGF1R) knockout mice. Compared with control littermates these mutants live longer without any obvious impairment of their health and physiology, except a reduced glucose tolerance that we observed in males. These IGF $1 \mathrm{R}^{+/-}$mutants were also more resistant to oxidative stress in vivo, and we identified a possible molecular pathway linking underphosphorylation of IGF-I receptors to the lack of activation of p66Shc, a protein capable of increasing resistance to oxidative stress through regulation of a set of downstream genes. These and other results suggest that in mammals too, lifespan can be increased by continuous, longterm downregulation of IGF signalling. Since growth hormone administration normally stimulates IGF production in tissues, the question arises whether the beneficial effects of $\mathrm{GH}$, as reported by others, could be IGF independent. This hypothesis can be addressed, for example, by adequately combining existing transgenic mouse models.
\end{abstract}

European Journal of Endocrinology 151 S23-S27

\section{Introduction}

Pioneering studies on lifespan regulation in rodents by McKay showed more than 70 years ago that life-long calorie restriction can significantly increase longevity, and these experiments have often been reproduced since (1). The underlying mechanism, although still not fully understood, also works in other mammalian species, and possibly in apes and humans. Other studies, using mutant mice with defects in pituitary development (Snell and Ames dwarf mice) (2, 3) revealed that panhypopituitarism with marked deficiency notably of thyrotrophin, prolactin and growth hormone $(\mathrm{GH})$ also leads to longevity. More recently, it was shown that the GH receptor itself, when inactivated by targeted mutagenesis, engenders significant extension of the mouse lifespan (4). Common to these quite different models is hypo- or infertility and a reduction in size and weight, which is also true for calorie restriction if started early in life. An additional trait of these models is the very low level of circulating insulin-like growth factor-I (IGF-I), an exciting finding since important genetic studies in Caenorhabditis elegans clearly identified insulin and insulin-like genes, namely daf-2, daf-16 and ins-7, as major regulators of the worm lifespan (5-8). Soon afterwards, it was shown that these evolutionary highly conserved insulin/insulin-like pathways also control the lifespan in yeast and insects $(9,10)$. The tyrosine kinase receptors transducing the insulin/insulin-like signals in these different species seem to play a major role in this process. For example, when levels of DAF-2 (the nematode homologue of the insulin and insulin-like receptors in mammals) are adequately diminished in C. elegans, the lifespan of the worm doubles. Interestingly, null mutants of daf- 2 will develop into a particularly durable larvae form of the worm, a process that can also be triggered when wild-type worms are confronted with very harsh environmental conditions and excessively high population density. In mice, however, insulin-like growth factor-I receptor (IGF1R) and insulin receptor (IR) null mutants die at birth or shortly thereafter, due to respiratory insufficiency and metabolic complications respectively (11). We were 
interested in studying the role of IGF signalling in mammalian longevity. Using IGF1R knockdown and knock out alleles in a gene dosage strategy, we generated mice with various degrees of IGF1R deficiency $(12,13)$. One of these, the heterozygous IGF1R mutant $\left(\mathrm{IGF} 1 \mathrm{R}^{+/-}\right.$), appeared appropriate for the study of the role of IGF1R in lifespan regulation in rodents (14). Results from this study will be summarized, extended and discussed in the following sections.

\section{Materials and methods}

Targeted mutations of the IGF1R gene were obtained by homologous recombination using a Cre-loxP mediated strategy (12). We targeted exon 3 of the IGF receptor gene, the exon which codes for most of the receptor ligand binding domain. Loss of exon 3 also leads to a premature stop during translation. Methods were described earlier and phenotypes of several IGF1R knockdown mutants were analysed in previous publications. Details of materials and methods can be found in Holzenberger et al. (13) or may be requested directly from the authors. Lifespan was determined in mouse cohorts comprising heterozygous mutants (IGF1R ${ }^{+/-}$) and their wild-type (WT) littermate control. Male as well as female cohorts were studied. They lived under standard conditions with free access to food and water. Ageing cohorts did not undergo any test or sampling except weighing every 2 months, and later a post-mortem histopathological analysis. All other phenotypic analyses were undertaken in additional, similarly structured experimental mouse cohorts. Briefly, blood samples from adult animals were used for routine biochemistry. IGF-I and insulin were measured using RIA-based assays purchased from DSL (Webster, Texas, USA) and Linco (rat sensitive insulin, Linco Res., St. Charles, Missouri, USA). Ligand binding assay of IGF1R was performed as previously described (15). Male and female fertility was measured as indicated in Holzenberger et al. (14). Metabolic rate was determined by indirect calorimetry (16) and glucose tolerance was tested using a handheld electronic device (Glucotouch, Lifescan, Johnson \& Johnson, Milpitas, California, USA). Resistance to oxidative stress was determined from individual resistance to a single intraperitoneal paraquat injection (17). Mouse embryonic fibroblast primary cultures were established individually from day 13.5 embryos and submitted to superoxide treatment in vitro. Activation of signal transduction pathways were studied using Western immunoblotting techniques described in detail by Dupont et al. (18).

\section{Results and discussion}

Receptor autoradiography and ligand binding assay in vitro showed that the cells of heterozygous IGF1R mutants $\left(\mathrm{IGF} 1 \mathrm{R}^{+/-}\right)$produced only half the normal number of receptors on their cells. Thus, loss of one IGF receptor allele does not seem to be compensated for by increased expression from the second, intact allele. RT-PCR analysis of IGF1R transcript prevalence showed that both alleles, the one lacking exon 3 as well as the WT allele, produced similar amounts of mRNA. We deduced from this that the mature transcripts of the mutant IGF1R gene are probably not subject to significant mRNA decay, as one could have expected from the fact that translation from this transcript prematurely stops in exon 4 due to a frame shift $(19,20)$.

The observed $50 \%$ reduction in receptor levels has only limited impact on the final size of $\mathrm{IGF}_{\mathrm{R}} \mathrm{R}^{+/-}$ mice (weight in young adult males: $-8 \%$; females: $-5 \%)$. Interestingly, the postnatal growth curves are identical for $\mathrm{WT}$ and IGF $1 \mathrm{R}^{+/-}$mice until weaning, and slight differences only developed thereafter. This is similar to our previous results (12), and is apparently typical for partial inhibition of IGF signalling in mice, as long as this inhibition does not go much beyond the $50 \%$ reduction in receptor levels. The impact of this IGF1R $\mathrm{R}^{+/-}$mutation on growth is more pronounced in males than in females, which has to be considered as a sex-dimorphic growth response. This particularity compares well with previous findings from our laboratory showing several sex-dimorphic responses after IGF receptor inactivation (12). We measured circulating IGF-I levels and found them significantly elevated ( $+32 \%$ on average), indicating an endocrine upregulation in response to IGF receptor insufficiency. This regulation was apparently insufficient to compensate for the initial loss of IGF1R.

Results from routine blood biochemistry in these mice indicated that IGF $1 \mathrm{R}^{+/-}$mutants were as healthy as their wild-type controls. Animals were observed until natural death occurred and their lifespan recorded. Survival analysis from these data revealed a significantly longer lifespan in IGF $1 \mathrm{R}^{+/-}$animals compared with wild-type controls, a tendency that was much stronger in females $(+33 \%, P<0.001)$ than in males $(+16 \%$, not significant). Results from necropsy suggested that in IGF1R $\mathrm{R}^{+/-}$mutants the spectrum of pathologies associated with death was comparable to wild-type populations, but the onset of pathologies seemed retarded. In a variety of models from different species used in ageing studies, low fertility has been found associated with increased longevity, and this relationship has been interpreted as an important trade-off between reproduction and survival function. Previous results obtained with insect models, however, suggested that IR/IGF1R mutations might produce longevous individuals without generating alterations in fertility, and our results can confirm this hypothesis: the onset of male and female fertility and the decline in female fertility were not different in IGF1R ${ }^{+/-}$mutants compared with WT mice. 
As mentioned above, calorie restriction is a very efficient means of increasing rodent lifespan, such that the observed positive effect on lifespan reported here could theoretically have been the result of dietary self restriction among IGF $1 \mathrm{R}^{+/-}$mice. To test this, long-term food and water intake were measured, and energy expenditure was determined individually. Nevertheless, this approach confirmed that longevous mutants and wild-type controls were indistinguishable in terms of food consumption and energy expenditure. Moreover, both groups showed highly similar circadian profiles of physical activity and body temperature. Together, this confirmed that IGF $1 \mathrm{R}^{+/-}$mice were healthy and that their physiology was normal in several respects. None of these results could, however, explain their longevity. Interestingly, we found that in males, but not in females, the glucose tolerance was reduced, while the capacity to secrete insulin was intact. This indicated that IGF $1 \mathrm{R}^{+/-}$males may suffer from a potential peripheral insulin resistance as a consequence of reduced IGF signalling. This finding is of considerable interest, given that the tonic secretion of insulin from the pancreatic $\beta$-cells itself depends on the presence of IGF receptors on the $\beta$-cells (21). It is at present unclear whether the potential long-term consequences of this pre-diabetic situation in IGF1 $\mathrm{R}^{+/-}$mice could have masked an otherwise possibly more important longevity among IGF1 $\mathrm{R}^{+/-}$males.

It is generally accepted today that oxidative stress is a major determinant of the ageing process by causing substantial damage to DNA, proteins and lipids, and finally to most or all cellular components. To find out whether alterations in the response to oxidative stress could explain, at least in part, the observed longevity, we submitted mutant mice and their controls to an intense intracellular oxidative stress that we induced by injection of methyl viologen. Clearly, mutants displayed increased resistance and survived significantly longer than wild-type controls $(P<0.05)$. Again, this effect was sex-dimorphic, being more pronounced in females than in males. To investigate whether this resistance was cell autonomous, we established mouse embryo fibroblast (MEF) cultures from IGFR $1^{+/-}$and control embryos and submitted these cell cultures to $\mathrm{H}_{2} \mathrm{O}_{2}$-induced oxidative stress in vitro. Such experiments revealed that cells with a reduced number of IGF receptors are better able to survive the oxidative stress. Although these results suggest that insulin/insulin-like signalling is able to modify the cell's resistance to oxidative stress, the molecular mechanism of this link is still largely unknown. Further experiments with MEFs, however, revealed that the two major intracellular pathways downstream of IGF1R were both less activated in the mutants, meaning that a reduction in receptor levels results in a broad downregulation of intracellular signal transduction. Of particular interest was the clear reduction of p66Shc phosphorylation, since this molecular isoform, a member of the Shc protein family, has recently been shown to regulate the resistance to oxidative stress in mice (17).

From the synopsis of these results we may further conclude that we need a better understanding of how the cells acquire their resistance to oxidative stress and which mechanisms and genetic programs exist in cells that allow them to enhance this stress resistance. One step forward in this process comes from the recent work of Murakami et al. (22) showing that fibroblasts from Snell dwarf mice retain their enhanced resistance to oxidative stress in vitro even after several rounds of cell culturing. This suggests that cells from these longevous dwarf mice must possess some form of cellular memory that allows them to differentially express genes implicated in the resistance of cells to oxidative stress and to continue to do so in culture. As a potential mechanism, Murakami et al. propose epigenetic changes that may occur while cells proliferate and differentiate in Snell dwarf mice with their particular hormonal context due to hypopituitarism. As potential targets for epigenetic changes, the authors mention genes of the insulin/insulin-like family, but interestingly also put forward p53 and retinoblastoma protein $(\mathrm{Rb})$, key molecules which could play major roles in regulating processes relative to lifespan in higher organisms. Further studies are needed to elucidate how these epigenetic changes are initiated. If we suppose that IGF1R deficiency plays a role in these processes then we could extract some information by analysing at which timepoint of development the inhibition of IGF signalling is most efficient in terms of regulation of growth and cell proliferation. We started to do so by establishing precise curves for the body weight gain in IGF1R-deficient mice. Figure 1 shows that for most of the postnatal development, growth velocity is identical for males and females of both IGF $1^{+/-}$and control genotypes, and that differences in growth velocity occur only during a very short period around the 3rd and 4 th week after birth. The inset shows that the curve around the velocity peak can indeed be distinguished into two distinct periods. At 3 weeks (solid arrow), the growth velocity curves split depending on whether the animals are IGF1R deficient or not. Shortly afterwards, a second peak (open arrow) occurs, where the growth velocity also differs but this time mainly depending on gender. The precise developmental timepoint when these peaks occur seems to depend also on the genetic background of the mice, but the phenomenon as such is reproducible on different backgrounds (not shown). Although these results are, of course, not direct proof, they suggest that significant changes in response to IGF1R deficiency occur during, and may in fact be limited to, short time periods. In addition, these findings suggest that growth velocity deficits due to lack of IGF1R may have some similitude with those changes in individual maturation that just several days afterwards generate the classical sex-related differences of somatic growth. 


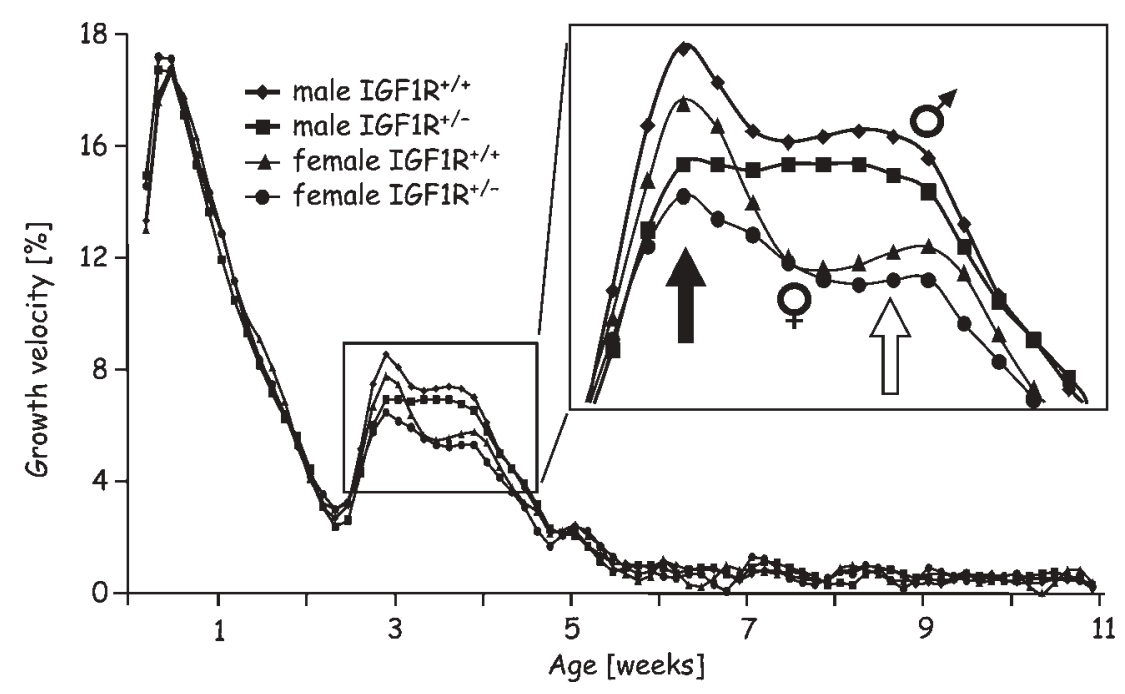

Figure 1 The growth velocity peak at 3 weeks of age is blunted in IGF1R ${ }^{+/-}$mutants. Growth velocity is weight gain per $24 \mathrm{~h}$ expressed as a percentage of absolute body weight. Curves were established using a sliding mean over 3 consecutive means.

One could speculate that both processes involve similar maturation processes and possibly the same elements of the somatotroph axis. It is of course tempting to go further and consider structures of the hypothalamic region and the pituitary gland as potential regulators of ageing in mammals. Another important question is the role of $\mathrm{GH}$ in the regulation of gene expression in peripheral tissues. This issue has recently been addressed by Ahluwalia et al. (23) who used a microarray approach to systematically examine the sexual dimorphism of liver gene expression in response to GH treatment. Their results suggest that GH-regulated gene expression is indeed the major determinant of sexually dimorphic gene expression in the rat liver. Similar mechanisms could help to explain how sexdimorphic development results from gender-specific pulsatile GH secretion pattern.

Also very interesting is the growing evidence that insulin/insulin-like signalling is linked to the regulation of resistance to oxidative stress in model organisms. Recently, a brilliant micro-array analysis by Murphy et al. (24) showed that a large number of stress-related genes are regulated by mutations in the nematode insulin-like genes, suggesting that the genetic link between insulin/insulin-like signals and stress resistance is quite broad and also very conserved during evolution. Others have made important contributions by putting these and other findings back into a larger perspective (see reference 25) and I would just like to reiterate that our present knowledge of lifespan regulation via somatotroph signals seems to indicate that changes in longevity can be most specifically induced by targeting IGFs or their receptor. IGF signalling can also be modified indirectly by targeting $\mathrm{GH}$, but the spectrum of induced phenotypic changes is then expected to be considerably broader. We conclude that more in-depth studies on the possible dissociation of GH and IGF actions are urgent, and that we need to understand, in more detail, how, on the one hand, the administration of GH can show beneficial effects on ageing and, on the other hand, how lowering IGF signalling can increase longevity. GH control over IGF expression is a relatively recent invention of evolution, and it is thus not excluded that we may gain additional valuable insights from a more evolutionary perspective.

\section{Acknowledgements}

M H is supported by grants from MENRT, GIS-Institut de la Longévité, INSERM ATC Vieillissement and La Ligue contre le Cancer.

\section{References}

1 Weindruch R, Walford RL, Fligiel S \& Guthrie D. The retardation of aging in mice by dietary restriction: longevity, cancer, immunity and lifetime energy intake. Journal of Nutrition 1986116 641-654.

2 Flurkey K, Papaconstantinou J, Miller RA \& Harrison DE. Lifespan extension and delayed immune and collagen aging in mutant mice with defects in growth hormone production. PNAS 2001 98 6736-6741.

3 Brown-Borg HM, Borg KE, Meliska CJ \& Bartke A. Dwarf mice and the ageing process. Nature $1996 \mathbf{3 8 4} 33$.

4 Coschigano KT, Clemmons D, Bellushi LL \& Kopchick JJ. Assessment of growth parameters and life span of GHR/BP gene-disrupted mice. Endocrinology $20001412608-2613$.

5 Kenyon C, Chang J, Gensch E, Rudner A \& Tabtiang R. A C. elegans mutant that lives twice as long as wild-type. Nature 1993 $366461-464$.

6 Guarente L \& Kenyon C. Genetic pathways that regulate ageing in model organisms. Nature $2000 \mathbf{4 0 8} 255-262$.

7 Kenyon C. A conserved regulatory system for aging. Cell 2001 $105165-168$. 
8 Gems D \& Partridge L. Insulin/IGF signalling and ageing: seeing the bigger picture. Current Opinion in Genetics and Development $200111287-292$.

9 Tatar M, Kopelman A, Epstein D, Tu MP, Yin CM \& Garofalo RS. A mutant Drosophila insulin receptor homolog that extends life-span and impairs neuroendocrine function. Science 2001292 107-110.

10 Bartke A. Mutations prolong life in flies: implications for aging in mammals. Trends in Endocrinology and Metabolism 200112 233-234.

11 Liu JP, Baker J, Perkins AS, Robertson EJ \& Efstratiadis A. Mice carrying null mutations of the genes encoding insulin-like growth factor I (Igf-1) and type 1 IGF receptor (Igf1r). Cell $19937559-72$.

12 Holzenberger M, Leneuve P, Hamard G, Ducos B, Perin L, Binoux M \& Le Bouc Y. A targeted partial invalidation of the IGF-I receptor gene in mice causes a postnatal growth deficit. Endocrinology $20001412557-2566$.

13 Holzenberger M, Hamard G, Zaoui R, Leneuve P, Ducos B, Beccavin C, Perin L \& Le Bouc Y. IGF-I receptor gene dosage generates a sexually dimorphic pattern of organ-specific growth deficits, affecting fat tissue in particular. Endocrinology 2001 $1424469-4478$.

14 Holzenberger M, Dupont J, Ducos B, Leneuve P, Geloen A, Even PC, Cervera P \& Le Bouc Y. IGF-I receptor regulates life span and resistance to oxidative stress in mice. Nature $2003 \mathbf{4 2 1}$ $182-187$.

15 Ducos B, Cabrol S, Houang M, Perin L, Holzenberger M \& Le Bouc Y. IGF type 1 receptor ligand binding characteristics are altered in a subgroup of children with intrauterine growth retardation. Journal of Clinical Endocrinology and Metabolism 200186 5516-5524.

16 Oudart H, Malan A, Maho Y \& Geloen A. Day-night pattern of energy expenditure and body temperature in cachectic tumour bearing rats. British Journal of Cancer 200083 1055-1060.
17 Migliaccio E, Giorgio M, Mele S, Pelicci G, Reboldi P, Pandolfi PP, Lanfrancone L \& Pelicci PG. The p66shc adaptor protein controls oxidative stress response and life span in mammals. Nature 1999 402 309-313.

18 Dupont J, Karas M \& LeRoith D. The potentiation of estrogen on insulin-like growth factor I action in MCF-7 human breast cancer cells includes cell cycle components. Journal of Biological Chemistry 2000275 35893-35901.

19 Pulak R \& Anderson P. mRNA surveillance by the Caenorabditis elegans smg genes. Genes and Development 19937 1885-1897.

20 Culbertson PMR \& Leeds F. Looking at mRNA decay pathways through the window of molecular evolution. Current Opinion in Genetics and Development 200313 207-214.

21 Kulkarni RN, Holzenberger M, Shih DQ, Ozcan U, Stoffel M, Magnuson MA \& Kahn CR. $\beta$-cell-specific deletion of the Igf-1 receptor leads to hyperinsulinemia and glucose intolerance but does not alter $\beta$-cell mass. Nature Genetics 200231 111-115.

22 Murakami S, Salmon A \& Miller RA. Multiplex stress resistance in cells from long-lived dwarf mice. FASEB Journal $2003 \mathbf{1 7}$ 1565-1566.

23 Ahluwalia A, Clodfelter KH \& Waxman DJ. Sexual dimorphism of rat liver gene expression: regulatory role of growth hormone revealed by deoxyribonucleic acid microarray analysis. Molecular Endocrinology 200418 747-760.

24 Murphy CT, McCarroll SA, Bargmann CI, Fraser A, Kamath RS, Ahringer J, Li JH \& Kenyon C. Genes that act downstream of DAF-16 to influence the lifespan of Caenorhabditis elegans. Nature $2003 \mathbf{4 2 4} 277-284$.

25 Lithgow GJ \& Gill MS. Cost-free longevity in mice? Nature 2003 421 125-126.

Received 24 March 2004

Accepted 3 April 2004 